Itinéraires Itinéraires

Littérature, textes, cultures

\title{
Erec R. Koch, The Æsthetic Body. Passion, Sensibility and Corporeality in Seventeenth-Century France
}

\section{Lucie Desjardins}

\section{OpenEdition}

\section{Journals}

Édition électronique

URL : http://journals.openedition.org/itineraires/414

DOI : 10.4000/itineraires.414

ISSN : 2427-920X

Éditeur

Pléiade

\section{Édition imprimée}

Date de publication : 1 mai 2009

Pagination : 194-196

ISBN : 978-2-296-08444-5

ISSN : $2100-1340$

\section{Référence électronique}

Lucie Desjardins, «Erec R. Koch, The AEsthetic Body. Passion, Sensibility and Corporeality in SeventeenthCentury France », Itinéraires [En ligne], 2009-1 | 2009, mis en ligne le 16 juin 2014, consulté le 22

septembre 2020. URL : http://journals.openedition.org/itineraires/414 ; DOI : https://doi.org/10.4000/ itineraires. 414

Ce document a été généré automatiquement le 22 septembre 2020.

\section{cc) $(1) \odot$}

Itinéraires est mis à disposition selon les termes de la licence Creative Commons Attribution - Pas d'Utilisation Commerciale - Pas de Modification 4.0 International. 


\title{
Erec R. Koch, The Æsthetic Body. Passion, Sensibility and Corporeality in Seventeenth-Century France
}

\author{
Lucie Desjardins
}

\section{RÉFÉRENCE}

Erec R. Koch, The Æsthetic Body. Passion, Sensibility and Corporeality in Seventeenth-Century

France, Newark, University of Delaware Press, 2008, 390 pages, ISBN : 978-0-874-13010-2

1 Au cours des dernières années, la place du corps au xviI siècle a fait l'objet de plusieurs travaux. On songe, bien sûr, au texte fondateur de Norbert Elias', qui a montré comment le "processus de civilisation » implique une contrainte s'exerçant sur les expressions du corps et comment les «bonnes manières » consistaient, dans une large mesure, en une intériorisation de ces contraintes; ou encore à Michel Foucault ${ }^{2}$, qui a entrepris de comprendre cette rationalisation des comportements à travers la notion de discipline. D'une tout autre manière, J.-J. Courtine et C. Haroche ${ }^{3}$ ont abordé, d'un point de vue historique, la question du corps signifiant réduit au seul visage tout en inscrivant ce dernier dans une histoire psychologique et sociale en posant les principaux jalons d'une étude du corps expressif. Plus récemment, A. Corbin, J.J. Courtine et G. Vigarello ${ }^{4}$ ont mis l'accent sur les usages du corps et de ses représentations en abordant des questions aussi diverses que celles de la médecine ou de la sexualité, du sacré ou de la vie en société.

2 Dans la poursuite de ces travaux sur le corps, mais aussi des approches contemporaines

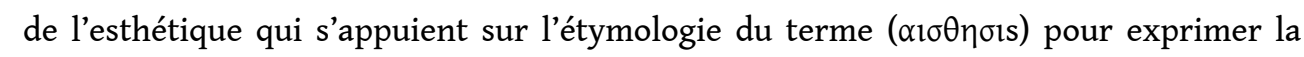
surdétermination de l'art par la sensation, Erec R. Koch propose ici une étude remarquable. À partir de ce qu'il appelle l'Æssthetic Body, expression qu'il faudrait sans doute traduire par corps sentant, puisqu'il est à la fois la source et le siège des passions 
et de la sensibilité, l'auteur propose d'examiner les transformations de la conception du corps au XVII ${ }^{\mathrm{e}}$ siècle.

3 Le premier chapitre est consacré à la physiologie depuis Vésale jusqu'à Descartes et aux cartésiens. Descartes, on le sait, tire toutes les conséquences philosophiques et morales du traité de Harvey sur la circulation sanguine dans son texte Les Passions de l'âme (1649). La passion, qui altère la couleur du visage en le faisant rougir ou pâlir, est ainsi expliquée par l'élargissement ou le resserrement des orifices du cœur. Si on retrouve plusieurs travaux sur Descartes et les passions, rares sont ceux qui ont analysé la correspondance du philosophe avec Élisabeth de Bohême qui, pourtant, constitue le point de départ de sa réflexion. Ici, l'auteur rétablit pleinement le rôle et l'importance de cette correspondance, tout en en soulignant l'élégance. Tout le chapitre s'emploie, du reste, à montrer de quelle façon les transformations de la médecine, de la physiologie et de la physique entraînent plusieurs ruptures épistémologiques.

4 Ces différentes ruptures sont illustrées par les quatre chapitres suivants. Le lien unissant le corps sentant aux passions y est alors développé à partir d'une analyse centrée sur quatre sens : la vue, l'ouïe, le goût, le toucher. Le chapitre sur la vue traite principalement de l'importance du spectacle et, par conséquent, de la vue, dans la dramaturgie de Corneille. C'est toutefois la description des processus à l'œuvre dans le phénomène de "contagion des passions " que l'on retrouve chez Pierre Nicole mais surtout chez Malebranche, qui retient l'attention. On rappellera, à la suite d'Erec R. Koch, que l'analyse malebranchienne définit la sympathie comme un effet de contagion des passions, qui sollicite surtout les mécanismes de l'imagination. De ce point de vue, la théorisation de l'imagination permet de prendre la mesure du déplacement de la question de la contagion des affects qui s'opère au tournant du siècle.

5 Le chapitre sur l'ouïe traite de la question de la voix à partir des traités de l'actio, qu'il s'agisse du Traitté de l'orateur, ou de la prononciation et du geste (1659) de Michel Le Faucheur ou encore de la Méthode pour bien prononcer un discours et le bien animer (1679) de René Bary. À la fois matérielle et immatérielle, la voix sert de lien entre le corps et les passions de l'âme, comme l'ont déjà, du reste, souligné les études qui font maintenant autorité. P.-J. Salazar ${ }^{5}$ et P. Dandrey ${ }^{6}$ se sont employés à mettre en évidence le rôle et la place qu'occupe cette question à l'âge classique en examinant des traités d'anatomie, de physique, d'acoustique et de rhétorique, et en montrant que la voix est un signe susceptible d'être observé et décodé, puis associé à une passion précise. Pour s'en aviser, il suffit d'ouvrir le Traitté de l'orateur, de Le Faucheur :

La Nature nous porte d'elle-mesme à prononcer autrement quand nous parlons de choses tristes \& lugubres, \& autrement quand nous parlons de choses joyeuses \& agréables. (Koch : 162)

6 Tout le XVII siècle cherche à montrer que, sous les sons et les mots, se dissimulent des passions que la voix exprime et représente. De même y aurait-il une voix naturelle qui se trouverait altérée par les passions et dont on cherchera constamment à rendre compte. Dans ce chapitre, Erec R. Koch examine ces convergences entre physiologie de l'ouïe (et de la voix) et théories des passions en centrant son analyse sur les textes de Marin Mersenne.

7 Le quatrième chapitre sur le goût est sans doute le plus intéressant. En effet, le goût est l'un des sens les moins étudiés depuis les travaux fondateurs de J.-L. Flandrin? Pourtant, et comme le souligne Erec R. Koch, le XvII siècle met en évidence les liens 
unissant goût et bon goût. Le goût est d'abord affaire de physiologie, mais aussi de passions, comme l'affirme Bernard Lamy dans La Rhétorique ou l'art de parler: "Les viandes qui remuent doucement les nerfs de la langue font ressentir à l'âme le plaisir de la douceur ; celles qui la coupent \& l'agitent avec violences sont aigres, piquantes, \& amères. » (Koch : 336)

Ici, la démonstration repose sur l'histoire culinaire et l'individuation du goût en examinant la manière dont le sens du goût qui caractérise les plaisirs de la table se déplace progressivement vers des normes visant à codifier le bon goût. Erec R. Koch convoque de nombreux textes, dont ce magnifique passage du Cuisinier roial et bourgeois (1691) de Massialot, qui affirme qu'en Europe « règne (sic) la propreté, le bon goût et l'adresse dans l'assaisonnement des viandes [...]» (p. 195). De ce point de vue, le bon goût constitue une expérience médiatisée du corps, du corps sentant, soumis aux passions.

Enfin, le dernier chapitre s'appuie sur Hobbes, Pierre Nicole et Pascal en étudiant la façon dont ces derniers utilisent des métaphores reliées au mouvement, à l'action, à l'impact ou au toucher pour décrire la vie civile, sociale et politique. On retiendra tout particulièrement la métaphore de Pierre Nicole, qui dépeint les mouvements de l'amour-propre en termes de forces physiques, de "tourbillons ", de mouvements, et même de collision (p. 235-236). Tout le chapitre s'emploie à mettre en lumière le lien entre le comportement, les mouvements mécaniques du corps sentant et l'expérience sensible des passions.

Si la question des passions a été souvent étudiée ces dernières années, je relèverai deux autres grandes qualités de ce texte. D'une part, il convient de souligner la place qu'accorde l'imposante bibliographie aux ouvrages anglo-saxons, qui sont souvent méconnus par la critique française. D'autre part, l'ouvrage adopte volontiers une démarche pluridisciplinaire en mettant en résonance plusieurs formes discursives. En tissant de façon exemplaire les liens qui unissent les différents sens aux traités philosophiques, textes médicaux, traités des passions, romans et pièces de théâtre, ouvrages d'art culinaire, etc., cet Aesthetic Body offre un nouvel éclairage sur l'histoire des rapports entre passions et corps sentant.

\section{NOTES}

1. Le Processus de civilisation, 1969.

2. Surveiller et Punir, 1975.

3. Histoire du visage. Exprimer et taire ses émotions, 1989.

4. Histoire du corps, 2005.

5. Le Culte de la voix au XVII siècle. Formes esthétiques de la parole à l'âge de l'imprimé, 1995.

6. «La Phoniscopie, c'est-à-dire la science de la voix », Littératures classiques. La voix au XVII éècle, 1990.

7. L'Ordre des mets, 2002. 


\section{AUTEURS}

\section{LUCIE DESJARDINS}

Université du Québec à Montréal, Canada 